\title{
ALMOST CONVERGENT AND ALMOST SUMMABLE SEQUENCES
}

\author{
PAUL SCHAEFER
}

1. Introduction. In [3], Lorentz introduced the notion of $F$-summability and discussed its relation to various summability methods defined by matrices. More recently, King [2] defined the concept of almost summability and almost regular matrices. In this paper we show that some of Lorentz's inclusion results for regular matrix methods can be extended to almost regular matrix methods.

2. Definitions. Let $m$ be the Banach space of real bounded sequences $x=\left\{x_{n}\right\}$ with the usual norm $\|x\|=\sup \left|x_{n}\right|$. There exist continuous linear functionals on $m$ called Banach limits [1]. It is well known that any Banach limit of $x$ lies between $\lim$ inf $x_{n}$ and $\lim \sup x_{n}$. An element $x$ of $m$ is said to be almost convergent, or $F$-summable, if all of its Banach limits are equal. We let $F$ denote the set of all almost convergent sequences and we write $F-\lim x=s$ if $s$ is the common value of all Banach limits of $x$. Lorentz [3] showed that $F-\lim x=s$ if and only if $\lim _{p}\left(x_{n}+\cdots+x_{n+p-1}\right) / p=s$ uniformly in $n$. He extended $F$-limits to bounded complex sequences by means of this condition.

Let $A=\left(a_{n k}\right)$ be a real infinite matrix. We say that $x$ is $F_{A}$-summable to $s$ if and only if $\lim _{n} \sum_{k} a_{n k} x_{k+p}=s$ uniformly in $p$. If $A(x)$ is the sequence $\left\{\sum_{k} a_{n k} x_{k}\right\}, x$ is said to be almost $A$-summable to $s$ if and only if $A(x)$ is in $F$ and $F$ - $\lim A(x)=s$. The matrix $A$ is said to be regular if $A(x)$ converges to $\lim x_{n}$ for all convergent sequences $x$. If $F-\lim A(x)=\lim x_{n}$ for all convergent sequences $x$, then the matrix $A$ is said to be almost regular. According to [2], a matrix $A$ is almost regular if and only if

(1) $\sum_{k}\left|a_{n k}\right|<H$ for all $n$,

(2) $a^{(k)}=\left\{a_{n k}\right\} \in F$ and $F$-lim $a^{(k)}=0$ for every $k$ and

(3) $a=\left\{\sum_{k} a_{n k}\right\} \in F$ and $F-\lim a=1$.

3. Inclusion theorems. Lorentz showed that if $A$ is a regular matrix and $x$ is $F_{A}$-summable to $s$, then $x$ is $F$-summable to $s$. A similar result holds for almost regular matrices.

THEOREM 1. If $A$ is almost regular and if $x$ is $F_{A}$-summable to $s$, then $x$ is F-summable to $s$.

Received by the editors April 14, 1967 and, in revised form, October 16, 1967. 
Proof. We paraphrase the proof of Theorem 2 of [3]. Let $x=\left\{x_{k}\right\}$ and $x^{(p)}=\left\{x_{k+p}\right\}$. Then $\left\|x^{(p)}\right\| \leqq\|x\|$ for every $p$. Set $y_{n p}=\sum_{k} a_{n k} x_{k+p}$ and suppose that $\lim _{n} y_{n p}=s$ uniformly in $p$. Then $y_{n p}=s+c_{n p}$, where given $\epsilon>0$ there is an $n_{0}>0$ so that $\left|c_{n p}\right|<\epsilon$ for all $n \geqq n_{0}$ and all $p$. As in Lorentz's proof, if we set $y^{(n)}=\left\{y_{n p}\right\}$, then $y^{(n)}=\sum_{k} a_{n k} x^{(k)}$ in the space $m$. Thus, if we set $c^{(n)}=\left\{c_{n p}\right\}$, we have $\sum_{k} a_{n k} x^{(k)}=s e+c^{(n)}$, where $e=\{1,1,1, \cdots\}$. If $B(x)$ denotes any Banach limit of $x$, then since $B(x)=B\left(x^{(k)}\right)$ for all $k$, and since Banach limits are continuous on $m$, we have for all $n \geqq n_{0}, \quad \sum_{k} a_{n k} B(x)=s+c_{n}$, where $\left|c_{n}\right|$ $=\left|B\left(c^{(n)}\right)\right|<\epsilon$. We now let $a=\left\{\sum_{k} a_{n k}\right\}$ and $c=\left\{c_{n}\right\}$. Then the last expression implies that $B(x) a=s e+c$. Once again take any Banach limit $B^{*}$ of both sides of this equation. Since the matrix $A$ satisfies (3) above, we get $B(x) B^{*}(a)=B(x) \cdot 1=s+B^{*}(c)=s$ since $\lim c_{n}=0$. Hence $x \in F$ and $F-\lim x=s$.

As has been observed by Lorentz, the $F$-summability method is relatively weak, in that $F$ is a subset of the convergence fields of many regular matrices. The next theorem generalizes Lorentz's result to almost regular matrices.

THEOREM 2. If $A$ is almost regular, if $F-\lim \left\{\sum_{k}\left|a_{n k}-a_{n, k+1}\right|\right\}=0$, then $A$ almost sums all almost convergent sequences. In fact, $F-\lim A(x)$ $=F-\lim x$ for every $x$ in $F$.

Proof. Let $x \in F$ and $F-\lim x=s$. Then, given $\epsilon>0$, there is a $p_{0}$ so that for all $p \geqq p_{0}$ and all $k,\left(x_{k}+\cdots+x_{k+p-1}\right) / p=s+b_{k p}$, where $\left|b_{k p}\right|<\epsilon / H, H$ being that of condition (1) for the almost regularity of $A$. Following Lorentz's proof of Theorem 7 in [3], we multiply by $a_{n k}$ and add, obtaining

$$
\frac{1}{p} \sum_{k} a_{n k}\left(x_{k}+\cdots+x_{k+p-1}\right)=s \sum_{k} a_{n k}+\sum_{k} a_{n k} b_{k p} .
$$

This can be rearranged to get

$$
\frac{1}{p} \sum_{k=0}^{p-2} \sum_{j=k}^{p-2} a_{n k} x_{j}+\frac{1}{p} \sum_{j=p-1}^{\infty} x_{j}\left(a_{n, j-p+1}+\cdots+a_{n j}\right) .
$$

Let $y_{n}=\sum_{k} a_{n k} x_{k}$. Then, upon combining these expressions, we get

$$
s \sum_{k} a_{n k}+\sum_{k} a_{n k} b_{k p}=y_{n}+A_{n p}+B_{n p}
$$

where

$$
A_{n p}=\sum_{k=0}^{p-2} x_{k}\left[\frac{1}{p} \sum_{j=0}^{k} a_{n j}-a_{n k}\right]
$$




$$
B_{n p}=\sum_{k=p-1}^{\infty} x_{k}\left[\left(a_{n, k-p+1}+\cdots+a_{n k}\right) / p-a_{n k}\right] .
$$

Set $a=\left\{\sum_{k} a_{n k}\right\}, A^{(p)}=\left\{A_{n p}\right\}, B^{(p)}=\left\{B_{n p}\right\}$, and $C^{(p)}=\left\{\sum_{k} a_{n k} b_{k p}\right\}$. Then in the space $m$, we have $s a+C^{(p)}=A(x)+A^{(p)}+B^{(p)}$.

For all $p \geqq p_{0},\left|\sum_{k} a_{n k} b_{k p}\right| \leqq \sum_{k}\left|a_{n k}\right| \cdot\left|b_{k p}\right|<\epsilon$ for all $n$. Hence, any Banach limit of $C^{(p)}$ cannot exceed $\epsilon$ in absolute value when $p \geqq p_{0}$.

If we denote the sequence $\left\{a_{n j}\right\}$ by $a^{(j)}$, we see that

$$
A^{(p)}=\sum_{k=0}^{p-2}\left[\frac{1}{p} \sum_{j=0}^{k} a^{(j)}-a^{(k)}\right] x_{k}
$$

and for any fixed $p$, any Banach limit of $A^{(p)}$ will be zero, since $F-\lim a^{(k)}=0$ for every $k$.

As in Lorentz's proof, we have the estimate

$$
\left|B_{n p}\right| \leqq[(p-1)\|x\| / 2] \cdot \sum_{k}\left|a_{n k}-a_{n, k+1}\right| \quad \text { for every } n .
$$

Since the $F$-limit of $\left\{\sum_{k}\left|a_{n k}-a_{n, k+1}\right|\right\}$ is zero, we see that any Banach limit of $B^{(p)}$ is zero. This follows because if $\left|s_{n}\right| \leqq\left|t_{n}\right|$ for every $n$, if $|t|=\left\{\left|t_{n}\right|\right\}$ and if $F$-lim $|t|=0$, it is easy to see that $F-\lim s=0$.

If we now take any Banach limit $B$ of the expression $s a+C^{(p)}$ $=A(x)+A^{(p)}+B^{(p)}$, we find that $|B(A(x))-s| \leqq \epsilon$ and $x$ is almost $A$-summable to $s$.

According to Theorem 7 of [3], $\lim _{n} \sum_{k}\left|a_{n k}-a_{n, k+1}\right|=0$ is both a necessary and sufficient condition that the regular matrix $A=\left(a_{n k}\right)$ sums all almost convergent sequences. The corresponding condition in Theorem 2 above for almost regular matrices turns out to be sufficient, but not necessary that an almost regular matrix almost sums all elements of $F$.

In order to show this, we use Lorentz's matrix, [3, p. 174]. Thus, let $\left\{a_{0}, a_{1}, \cdots\right\}$ be a real sequence such that $\sum_{k} a_{k}=1, \sum_{k}\left|a_{k}\right|$ $<+\infty$, and $a_{0} \neq a_{1}$. Define the matrix $A=\left(a_{n k}\right)$ as $a_{n k}=a_{k-n}$ if $k \geqq n$, $a_{n k}=0$ otherwise. Then $A$ is a regular matrix, and hence is almost regular. For any $x \in F, A_{n}(x)=\sum_{k} a_{n k} x_{k}=\sum_{k=0}^{\infty} a_{k} x_{n+k}$, and $\left[A_{n}(x)+\cdots+A_{n+p-1}(x)\right] / p=\sum_{k=0}^{\infty} a_{k}\left[x_{n+k}+\cdots+x_{n+k+p-1}\right] / p$. Suppose that $F-\lim x=s$. Then, given $\epsilon>0$, there is a $p_{0}>0$ so that for all $p>p_{0}$ and all $n,\left[x_{n}+\cdots+x_{n+p-1}\right] / p=s+e_{n p}$, where $\left|e_{n p}\right|$ $<\epsilon / H, H$ denoting $\sum_{k}\left|a_{k}\right|$. Now, $\left[A_{n}(x)+\cdots+A_{n+p-1}(x)\right] / p$ 
$=\sum_{\mathbf{t}=0}^{\infty} a_{k}\left(s+e_{n+k, p}\right)=s+f_{n p}$, where $\left|f_{n p}\right|<\epsilon$ for all $p>p_{0}$ and all $n$. Hence, $F-\lim A(x)=s$, although we have $\sum_{k}\left|a_{n k}-a_{n, k+1}\right| \geqq\left|a_{0}-a_{1}\right|$ $>0$ for every $n$.

\section{REFERENCES}

1. C. Goffman and G. Pedrick, First course in functional analysis, Prentice-Hall, Englewood Cliffs, N. J., 1965.

2. J. P. King, Almost summable sequences, Proc. Amer. Math. Soc. 17 (1966), 1219-1225.

3. G. G. Lorentz, A contribution to the theory of divergent sequences, Acta Math. 80 (1948), 167-190.

State University College, Geneseo 